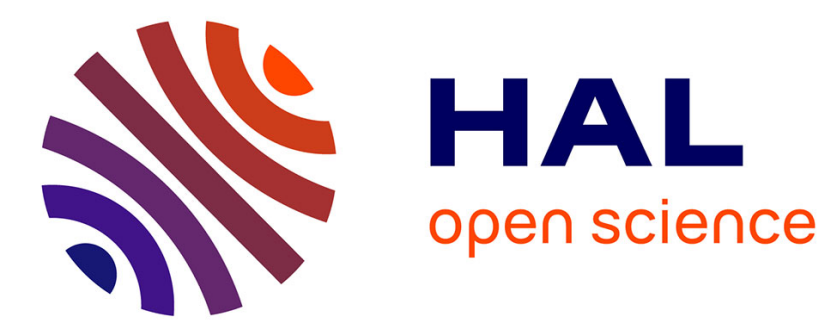

\title{
Using Fourier-based shape alignment to add geometric prior to snakes
}

Mohamed-Ali Chermi, Faouzi Ghorbel, Stéphane Derrode

\section{To cite this version:}

Mohamed-Ali Chermi, Faouzi Ghorbel, Stéphane Derrode. Using Fourier-based shape alignment to add geometric prior to snakes. ICASSP'09, Apr 2009, Taipei, Taiwan. hal-00437091

\section{HAL Id: hal-00437091 \\ https://hal.science/hal-00437091}

Submitted on 29 Nov 2009

HAL is a multi-disciplinary open access archive for the deposit and dissemination of scientific research documents, whether they are published or not. The documents may come from teaching and research institutions in France or abroad, or from public or private research centers.
L'archive ouverte pluridisciplinaire HAL, est destinée au dépôt et à la diffusion de documents scientifiques de niveau recherche, publiés ou non, émanant des établissements d'enseignement et de recherche français ou étrangers, des laboratoires publics ou privés. 


\section{USING FOURIER-BASED SHAPE ALIGNMENT TO ADD GEOMETRIC PRIOR TO SNAKES}

Mohamed Ali Charmi, Faouzi Ghorbel

\author{
Pôle GRIFT, Laboratoire CRISTAL, ENSI \\ Campus Universitaire de la Manouba \\ Tn-2010 Manouba, Tunisia. \\ charmi.madfree.fr \\ faouzi.ghorbel@ensi.rnu.tn
}

\author{
Stéphane Derrode \\ Institut Fresnel (CNRS UMR 6133), ECM, \\ Technopôle de Château-Gombert, \\ 8, rue Frédéric Joliot Curie, \\ 13451 Marseille Cedex 20, France. \\ stephane. derrodedfresnel. fr
}

\begin{abstract}
In this paper, we present a new algorithm of snakes with geometric prior. A method of shape alignment using Fourier coefficients is introduced to estimate the Euclidean transformation between the evolving snake and a template of the searched object. This allows the definition of a new field of forces making the evolving snake to have a shape similar to the template one. Furthermore, this strategy can be used to manage several possible templates by computing a shape distance to select the best one at each iteration. The new method also solves some well-known limitations of snakes such as evolution in concave boundaries, and enhances the robustness to noise and partially occluded objects. A series of experimental results is presented to illustrate performances.
\end{abstract}

Index Terms - Snakes, geometric prior, Euclidean Transform, Alignment.

\section{INTRODUCTION}

Active contours [1] are iterative methods of edge detection by means of energy minimization. Many variants of active contours have been presented, among them parametric models (snakes [1]), level sets [2], and geodesic active contours [3]. The active contours evolution is driven essentially by pixel values which are low level primitives. Therefore, original algorithms show some limitations in presence of noise, occlusions and clutters. Especially, the snakes model suffers from its sensitiveness to initial guess and its disability to evolve in concave boundaries.

A way to cope with active contours limitations is to introduce prior information about the shape of the object to be retrieved. The prior information can be represented by a drawn sketch, another instance of the object or a trained data. Many approaches have been presented recently that we can roughly divide in two categories: those which assume statistical shape prior and those which make use of geometrical ones. In the case of geometric shape prior, paper [4]proposes to minimize

a distance between the region inside the evolving contour and the template. This distance is computed between the Legendre moments, which ensures invariance to translation, rotation and scaling. In [5], we propose to add shape prior to parametric active contours using Fourier-based invariants to Euclidean transformations. Statistical shape prior can also be added to active contours. In [6], Staib \& al. determine the parameters of a Gaussian probability distribution that associates the object boundaries to a range of shapes. The optimization problem is then performed by the maximum a posteriori using Bayesian rule. Eigensnake [7] are driven by an affineinvariant deformable contour. The internal energy is used to define the global and local shape deformations between the shape and the image domains in a Bayesian framework. Diffusion snakes [8] are a method of snakes with statistical shape prior using a modified Mumford-Shah functional.

This work aims at adding a geometrical shape prior to snakes by aligning the evolving snake and the template. Transformation parameters are estimated thanks to the Hausdorff distance, expressed in the Fourier domain. The outlines of the shape alignment method are presented in Section 2. Section 3 describes the way a shape prior is introduce to the snakes model through shape alignment using Fourier coefficients of closed contours. Experimental results are commented in Section 4. Section 5 concludes the work and highlight some possible perspectives.

\section{SHAPE ALIGNMENT USING FOURIER COEF.}

The closed contour of a planar object can be represented by a parametric equation $\gamma:[0,2 \pi] \longrightarrow \mathbb{C}$

$$
l \longmapsto x(l)+i y(l),
$$

with $i^{2}=-1$. For latter use, the Fourier coefficients of $\gamma$ is given by

$$
C_{k}(\gamma)=\int_{0}^{2 \pi} \gamma(l) e^{-i k l} d l, \quad k \in \mathbb{Z}
$$


Now let $\gamma_{1}$ and $\gamma_{2}$ be centered (according to the center of mass) and normalized arclength parameterizations of two closed planar curves having shapes $F_{1}$ and $F_{2}$. Hence, scale factor and translation between the two curves can be ignored.

Ghorbel [9] shown that the following quantity is a metric between shapes:

$$
d\left(F_{1}, F_{2}\right)=\inf _{\left(l_{0}, \theta\right) \in T^{2}}\left\|\gamma_{1}(l)-e^{i \theta} \gamma_{2}\left(l+l_{0}\right)\right\|,
$$

where $T=[0,2 \pi]$ is the range of the rotation angle $\theta$, and of the difference between the starting description points for the two curves $l_{0}$. By using the shift theorem in the Fourier domain, computing such a distance comes down to the minimization of

$$
f\left(\theta, l_{0}\right)=\sum_{k \in \mathbb{Z}}\left|C_{k}\left(\gamma_{1}\right)-e^{i\left(k l_{0}+\theta\right)} C_{k}\left(\gamma_{2}\right)\right|^{2} .
$$

Persoon \& al. [10] proposed a solution to compute $l_{0}$ and $\theta$. First, $l_{0}$ is one of the zeros of the function

$$
\begin{array}{r}
g(l)=\sum_{k} \rho_{k} \sin \left(\psi_{k}+k l\right) \sum_{k} k \rho_{k} \cos \left(\psi_{k}+k l\right)- \\
\sum_{k} k \rho_{k} \sin \left(\psi_{k}+k l\right) \sum_{k} \rho_{k} \cos \left(\psi_{k}+k l\right),
\end{array}
$$

where $\rho_{k} e^{i \psi_{k}}=C_{k}^{*}\left(\gamma_{1}\right) C_{k}\left(\gamma_{2}\right)$. Second, $\theta$ is chosen to satisfy (6) and minimize $f\left(\theta, l_{0}\right)$ where $l_{0}$ is one of the roots of (5).

$$
\tan \theta=-\frac{\sum_{k} \rho_{k} \sin \left(\psi_{k}+k l_{0}\right)}{\sum_{k} \rho_{k} \cos \left(\psi_{k}+k l_{0}\right)} .
$$

Ghorbel proved in [9] the uniqueness of the parameters so obtained. Indeed, minimizing (4) is equivalent to compute the Hausdorff distance between shapes in the Fourier domain. An example of alignment between two contours with the same shape but distinct parameters $\theta$ and $l_{0}$ is given in Fig. 1 .

\section{SHAPE PRIOR EMBEDDING IN SNAKES}

We start by recalling the principles of parametric active contours. Then we describe how to add shape prior to the model using contours alignment between the template and the evolving curve. We end with the case of several possible templates.

\subsection{Snakes Model}

A snake [1] is a parameterized curve $v(l, t)$ moving under internal and external energies to reach features of interest in the image such as edges. The internal energy corresponds to the first and second derivatives of $v(l, t)$ and aims at keeping the curve smooth and preventing the appearance of corners. The external energy is computed on the image gray-levels and attracts the curve to strong gradients. The energy of the snakes

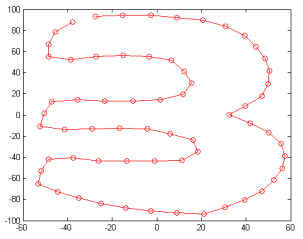

(a)

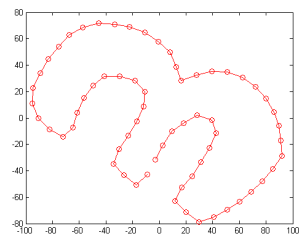

(b)

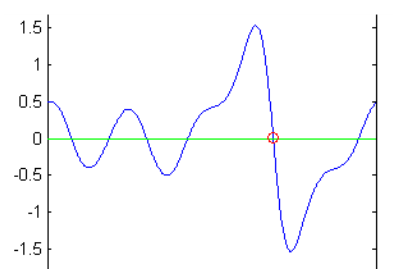

(c)

Fig. 1. Example of shape alignment using Fourier Descriptors: (a) and (b) are the two curves; (c) is the plot of $g(l)$. The $l_{0}$ value minimizing $f$ is plot in red.

is given by:

$$
\begin{array}{r}
E(v(l, t))=\int_{0}^{1} w_{1}\left|v^{\prime}(l, t)\right|^{2}+w_{2}\left|v^{\prime \prime}(l, t)\right|^{2} \\
-w_{3}\left|\nabla\left(G_{\sigma} * I\right)\right|^{2} d l .
\end{array}
$$

Coefficients $w_{1}, w_{2}$ and $w_{3}$ weight the different energies. Their value is set experimentally.

The snakes equation was first solved by Kass \& al. using Euler-Lagrange framework, which gives, after discretization

$$
\left(I_{N}+\tau \mathbf{A}\right) v(t)=v(t-1)+\tau F_{e x t}(v(t-1)),
$$

where $\mathbf{A}$ denotes the stiffness matrix, representing all internal elasticity relations of the snake, which is pentadiagonal and symmetric. $\tau$ denotes the time step, $N$ the number of nodes of the snakes, $I_{N}$ the identity matrix and $F_{\text {ext }}$ the forces derived from the external energy:

$$
F_{\text {ext }}=-\nabla\left|\nabla\left(G_{\sigma} * I\right)\right|^{2} .
$$

\subsection{Adding Shape Prior to the Model of Snakes}

At each iteration $t$, parameters $l_{0}$ and $\theta$ are estimated between the snakes and the template, using the algorithm described in Section 2. The two curves do not have the same shape but the two estimated parameters correspond to the "best" transformation to align one shape with the other. That way, we have a correspondance between each node of the snake and each node of the template, assuming the two curves are sampled with the same number of nodes $N$.

Hence, it becomes possible to compute an Euclidean distance between each corresponding nodes, and to add new forces which are weighted by the distance between the two 
curves. These forces make the evolving snakes to have the same shape than the template.

Let $v^{t}$ represents the template shape. Once $l_{0}$ and $\theta$ are retrieved, we can align the two shapes to obtain $v_{r}^{t}$ where the DFT of $v_{r}^{t}$ is given by

$$
C_{k}\left(v_{r}^{t}\right)=e^{-i \theta} e^{i k l_{0}} C_{k}\left(v^{t}\right)
$$

External energy remains the principal energy of the model ; the prior forces act especially in the absence of this energy. This situation can occur in presence of occlusions or at the entrance of a concave boundary. If we denote

$$
F_{\text {prior }}=\frac{v_{r}^{t}(l, t)-v(l, t)}{\left|v_{r}^{t}(l, t)-v(l, t)\right|}
$$

the new force of the snakes becomes :

$$
F_{\text {snakes }}=c_{1} F_{\text {prior }}+c_{2} F_{\text {ext }}
$$

with $c_{1}$ and $c_{2}$ two constant weights set experimentally (a bigger importance is given to gradient-driven energy). Internal energies do not appear in eq. (12) since they are already included in matrix $A$, see eq. (8).

\subsection{Case of Multiple Templates}

In presence of many templates, we propose to choose iteratively the most suitable one using as criterion the distance between the set of invariants of the evolving snake and the one of available templates. We use the complete and stable set of invariant Fourier descriptors introduced by Ghorbel in [9]:

$$
\begin{aligned}
I_{k_{0}}(\gamma)= & \left|C_{k_{0}}(\gamma)\right|, \quad \text { for } k_{0} \text { such that } C_{k_{0}}(\gamma) \neq 0 \\
I_{k_{1}}(\gamma)= & \left|C_{k_{1}}(\gamma)\right|, \quad \text { for } k_{1} \neq k_{0} \text { such that } C_{k_{1}}(\gamma) \neq 0 \\
I_{k}(\gamma)= & \frac{C_{k}(\gamma)^{k_{0}-k_{1}} C_{k_{0}}(\gamma)^{k-k_{1}} C_{k_{1}}(\gamma)^{k_{0}-k}}{I_{k_{0}}(\gamma)^{k-k_{1}-p} I_{k_{1}}(\gamma)^{k_{0}-k-q}} \\
& \forall k \neq k_{0}, k_{1}, \text { with } p, q>0 .
\end{aligned}
$$

This set is invariant to desired transformations, especially rotation and starting description point of the curve. The stability property of the set ensures that a slight modification of invariants does not induce a noticeable shape distortion, which is interesting for the stability of the snakes. To compare the snakes and templates, we use the following distance:

$$
d\left(\gamma_{1}, \gamma_{2}\right)=\left(\sum_{k}\left|I_{k}\left(\gamma_{1}\right)-I_{k}\left(\gamma_{2}\right)\right|^{\frac{1}{2}}\right)^{2}
$$

Giving many templates, we compute the invariants of the snakes and all the available shapes according to eq. (13). Then, we compute the distance between the invariants of the snake and each shape according to eq. (14). The shape having the minimum distance to the snake is used as template.

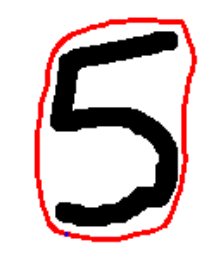

(a) Initialisation

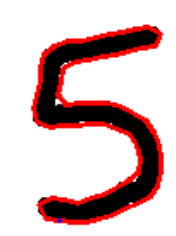

(b) Result
Fig. 2. Result on a shape with two deep concavities.

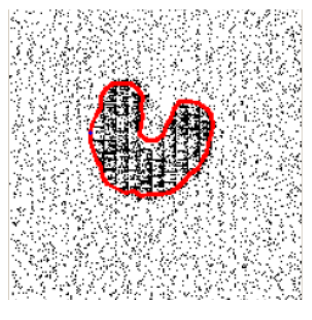

(a) Noisy image

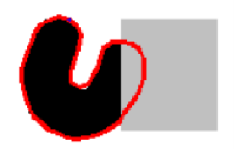

(b) Partly occluded object
Fig. 3. Results of the proposed method on the $U$ form when confronted to noise (a) and to partly occluded objects (b).

\section{EXPERIMENTAL RESULTS}

In this section, we present a set of experimental results and discuss parameter effects. The method is then applied on myocardial scintigraphy images.

The classical snakes model is not able to evolve in concavities due to absence of forces at the entrance of the concavity. Only a few models coping with this problem have been presented such as GVF [11], but their success depends on the concavity deepness. Our model is able to cope with such concavities, as illustrated in Fig. 2.

We also test our method robustness to noise and to partial occlusion, some results are reported in Fig. 3. Despite an high noise rate (a), the $U$ form is well detected. The method also succeeds to find the desired shape in presence of partial occlusion (b).

To illustrate the influence of the prior force only, Fig. 4 exhibits some iterations of our method on the 5 digit, when

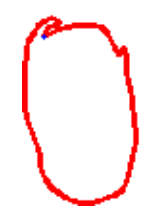

(a)

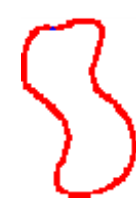

(b)

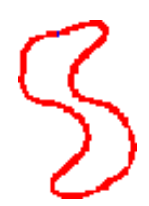

(c)

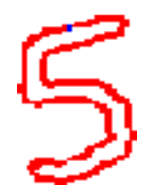

(d)
Fig. 4. Some iterations of our model under the prior forces $F_{\text {prior }}$ only. 


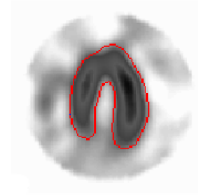

(a)

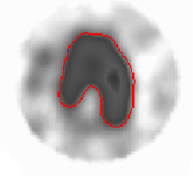

(b)

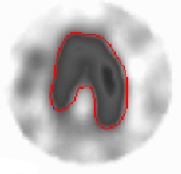

(c)

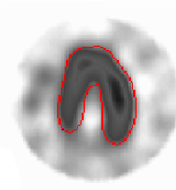

(d)
Fig. 5. Application of the method to the segmentation of four myocardial scintigraphy slices.

using Fig. 4(d) as a template. As expected, the curve evolves iteratively to produce the same shape than the template.

Compared to classical snakes methods [1], our method give better results, especially for noisy image and partly occluded object. Indeed, prior forces help the snakes to escape noisy points having strong gradients and making the final result not similar to template. In addition, in absence of gradient (concave boundary and partly occluded objects), the snake is guided by prior forces which makes the snake to have the same shape than the proposed template. We also compared our method with a recent method of snakes with shape prior [5] using Fourier invariants and we get better results in presence of noise and occlusion.

The complexity of our method is higher than the algorithm of Kass \& al. but remains lower than level set methods [2]. At each iteration, we compute the FFT of the evolving snake and estimate the transformation parameters. To reduce the complexity of parameters estimation we can truncate the Fourier coefficients. Indeed, it has been experimented in [9] that, for relatively smooth contours, 20 Fourier coefficients are sufficient enough to get a good approximation of $\theta$ and $l_{0}$. In addition, at advanced stages of the iterative algorithm, the parameters estimation becomes more reliable and we can reduce the search space.

We applied our method to the segmentation of myocardial scintigraphy images. As template, we used a sketch representing a shape similar to the structure to be detected. The initial snakes position was set around the myocarduim. The segmentation results are shown in Fig. 5 and seem visually satisfactory. To take into account pathological cases, we can automatically reduce the influence of the prior force (coefficient $c_{1}$ in eq. (12)) as the snake converges, increasing the influence of image data on the snakes.

\section{CONCLUSION \& PERSPECTIVES}

A new method of snakes with shape prior has been presented. A shape alignment method is used the estimate the transformation (rotation angle and starting point) between the snake and template. Parameters estimation is performed by minimizing the Hausdorff distance in the Fourier domain. This allowed the introduction of a new force that moves the snake to have the same shape than the provided template. Experimental results show the method ability to make snake evolve in deep concave boundaries. In addition, partially occluded objects can be detected. We now plan to apply the method to level set methods assuming that the image contains only one object and extend the prior to affine transformations [12] which are more general than the Euclidean ones.

\section{REFERENCES}

[1] M. Kass, A. Witkin, and D. Terzopoulos, "Snakes: active contour models," Int. J. of Comp. Vis., vol. 1, no. 4, pp. 321-331, 1988.

[2] S. Osher and J. A. Sethian, "Fronts propagating with curvature-dependent speed: algorithms based on Hamilton-Jacobi formulations," J. of Computational Physics, vol. 79, pp. 12-49, 1988.

[3] V. Caselles, R. Kimmel, and G. Sapiro, "Geodesic active contours," Int. J. of Comp. Vis., vol. 22(1), pp. 61-79, 1997.

[4] A. Foulonneau, P. Charbonnier, and F. Heitz, "Affineinvariant geometric shape priors for region-based active contours," IEEE trans. PAMI, vol. 28, no. 8, pp. 13521357, 2006.

[5] M. A. Charmi, S. Derrode, and F. Ghorbel, "Fourierbased shape prior for snakes," Pat. Recog. Let., vol. 29(7), pp. 897-904, 2008.

[6] L.H. Staib and J.S. Duncan, "Boundary finding with parametrically deformable models," IEEE trans. PAMI, vol. 14, no. 11, pp. 1061-1075, 1992.

[7] Z. Xue, S. Li, and E. Teoh, "Ai-eigensnake: An affineinvariant deformable contour model for object matching," Im. Vis. Comp., vol. 20, no. 2, pp. 784-2002, 2002.

[8] D. Cremers, F. Tischhauser, J. Weickert, and C. Schnorr, "Diffusion snakes: introducing statistical shape knowledge into the Mumford-Shah functional," Int. J. of Comp. Vis., vol. 50, pp. 295-313, 2002.

[9] F. Ghorbel, "Towards a unitary formulation for invariant image description: application to image coding," An. of telecom., vol. 153, no. 3, pp. 145-155, 1998.

[10] E. Persoon and K. S. Fu, "Shape discrimination using Fourier descriptors," IEEE trans. PAMI, vol. 8, no. 3, pp. 388-397, 1986.

[11] C. Xu and J. Prince, "Snakes, shapes, and gradient vector flow," IEEE trans. IP, vol. 7(3), pp. 359-369, 1998.

[12] F. Chaker, M. T. Bannour, and F. Ghorbel, "Contour retrieval and matching by affine invariant Fourier descriptors," in IAPR conf. on MVA, Tokyo, May 16-18, 2007. 